\title{
A Less Virulent COVID-19 Pneumonia
}

\author{
Andrew George ${ }^{1}$, Latha Ganti 2, 3, 4 \\ 1. Emergency Medicine, Brown University, Providence, USA 2. Emergency Medicine, Envision Physician Services, \\ Plantation, USA 3. Emergency Medicine, University of Central Florida College of Medicine, Orlando, USA 4. Emergency \\ Medical Services, Polk County Fire Rescue, Bartow, USA
}

Corresponding author: Latha Ganti, latha.ganti@ucf.edu

\begin{abstract}
Since its initial outbreak, COVID-19 saw a high death rate with those infected typically presenting with severe respiratory distress along with multi-organ involvement. However, over the course of the pandemic, particularly due to the lower age of those diagnosed with the disease and a greater understanding of the risk posed to certain at-risk populations, a new disease course seems to be more prominent with an overall lower mortality among those diagnosed. We present a typical example of such a case here, showing a less lethal course of COVID-19 occurring in late June amidst the resurgence of new daily cases in the United States.
\end{abstract}

Categories: Emergency Medicine, Infectious Disease

Keywords: covid-19, pneumonia

\section{Introduction}

The novel coronavirus, severe acute respiratory syndrome coronavirus 2 (SARS-CoV-2), outbreak responsible for COVID-19 quickly moved to international significance from the initial cases in Wuhan, reported to the World Health Organization on December 31 [1]. By the end of the next month, 9,976 cases were reported over 21 countries, with the first US case being reported on January 20 [2]. As the situation developed over the next couple months, various measures taken in the United States - including enforced quarantines, cancellations of social events, and other precautions - reduced the number of new cases per day from a high of 43,438 on April 6 to a low of 14,676 on June 3 [3]. State "re-openings" throughout mid-to-late June into July appear to have triggered a new resurgence in new cases, with around 55,000 new cases daily in early July $[4]$.

However, data suggest that for a variety of reasons, the course of typical COVID-19 outcomes in the most recent month differs from when the virus first came to the country. With the average age of diagnosed patients decreasing, the ratio of deaths to cases appears to be dropping, with even recent hospital visits resulting in discharge after a brief stay in the emergency department (ED) [5].

Received 07/16/2020 Review began 07/18/2020 Review ended 07/20/2020 Published 07/27/2020

(c) Copyright 2020 George et al. This is an open access article distributed under the terms of the Creative Commons Attribution License CC-BY 4.0., which permits unrestricted use, distribution, and reproduction in any medium, provided the original author and source are credited.
We present one such case of a late middle-age male presenting to the ED with a case of COVID-19 pneumonia but being well enough to go home from the ED. Such cases are becoming more common in this resurgence of COVID-19 and may very well represent the new expected course of the disease. Moreover, the use of CT in this case is typical of a practical means of diagnosing COVID-19 in emergency settings, where traditional real-time polymerase chain reaction (RT-PCR) tests may be unavailable [6].

\section{Case Presentation}

In late June, a 51-year-old male presented to the ED with a chief complaint of shortness of breath. The patient reported sporadic fevers and chills at home over the past two weeks, but denied current fever, nausea, vomiting, diarrhea, abdominal pain, headache, or any urinary symptoms. The patient reported no past medical history and was not on any medications. His son, however, was diagnosed with COVID-19 two weeks prior, with a cough, fever, and typical viral syndrome type symptoms. The patient reported that he did not quarantine from his son even after the positive COVID-19 diagnosis, as they lived in the same house.

The patient's vital signs were temperature $99.1^{\circ} \mathrm{F}$, blood pressure $111 / 76 \mathrm{mmHg}$, pulse rate 87 beats per minute, respiration rate 18 breaths per minute, and oxygen saturation of $94 \%$ on room air. Physical examination revealed significantly decreased breath sounds bilaterally. The remainder of physical examination was unremarkable, including cardiovascular and neurological examination. Laboratory analysis showed signs of leukopenia (white blood cell count of 3,000 per cubic $\mathrm{mm}$ ) but was negative for neutrophilia or lymphocytopenia. All electrolytes were within normal limits. The D-dimer count was elevated at 2.05 $\mathrm{mg} / \mathrm{L}$. Radiological examination included a chest $\mathrm{x}$-ray that demonstrated scattered opacities throughout both lungs suggestive of multifocal atypical pneumonia (Figure 1). 


\section{Cureus}

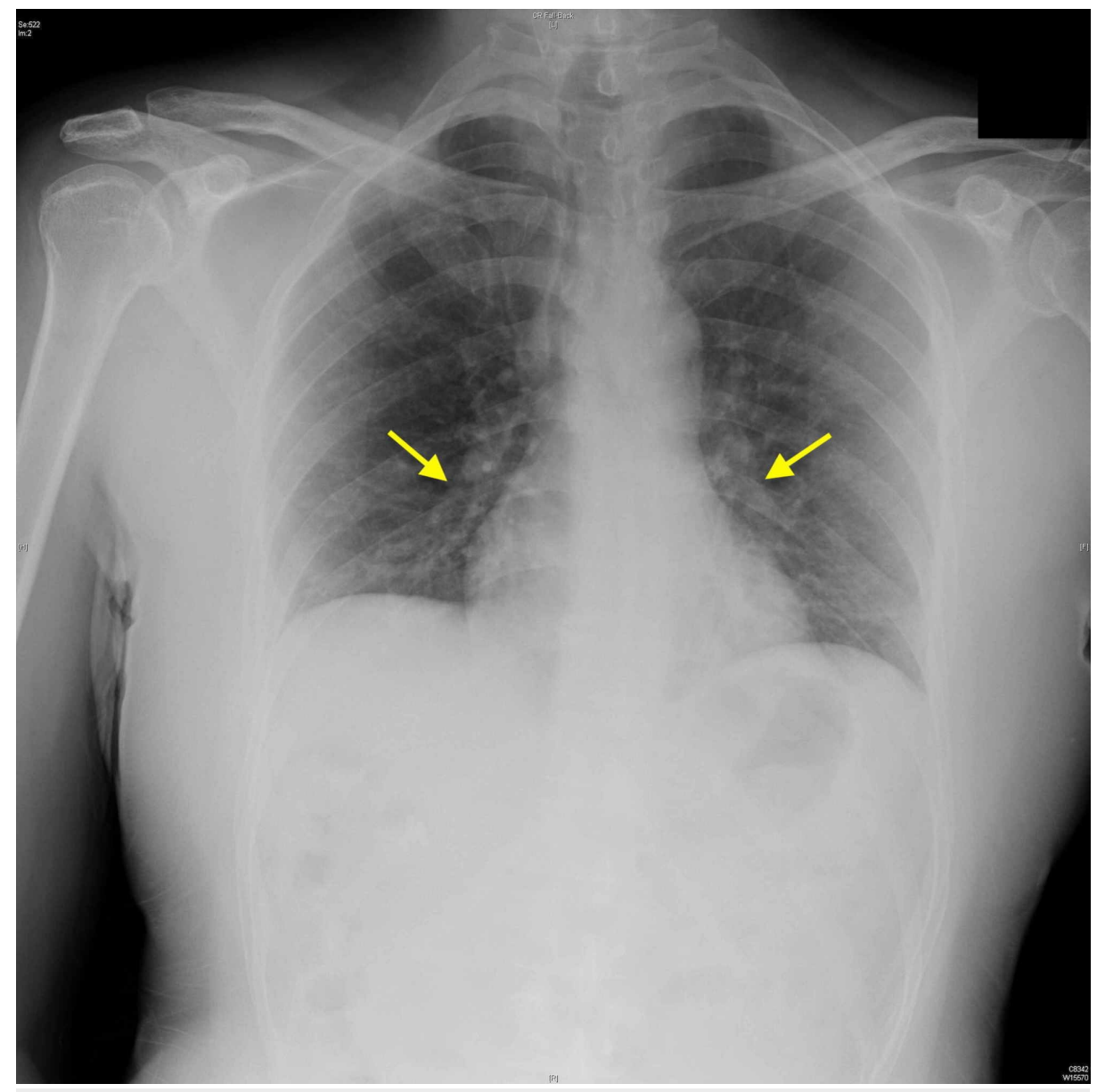

FIGURE 1: Chest radiograph demonstrating bilateral opacities associated with multifocal pneumonia (arrows)

Chest CT showed multifocal ground glass opacities (GGO) throughout both lungs typical of underlying SARS-CoV-2 infection. There was no evidence for pulmonary embolism (Figure 2).

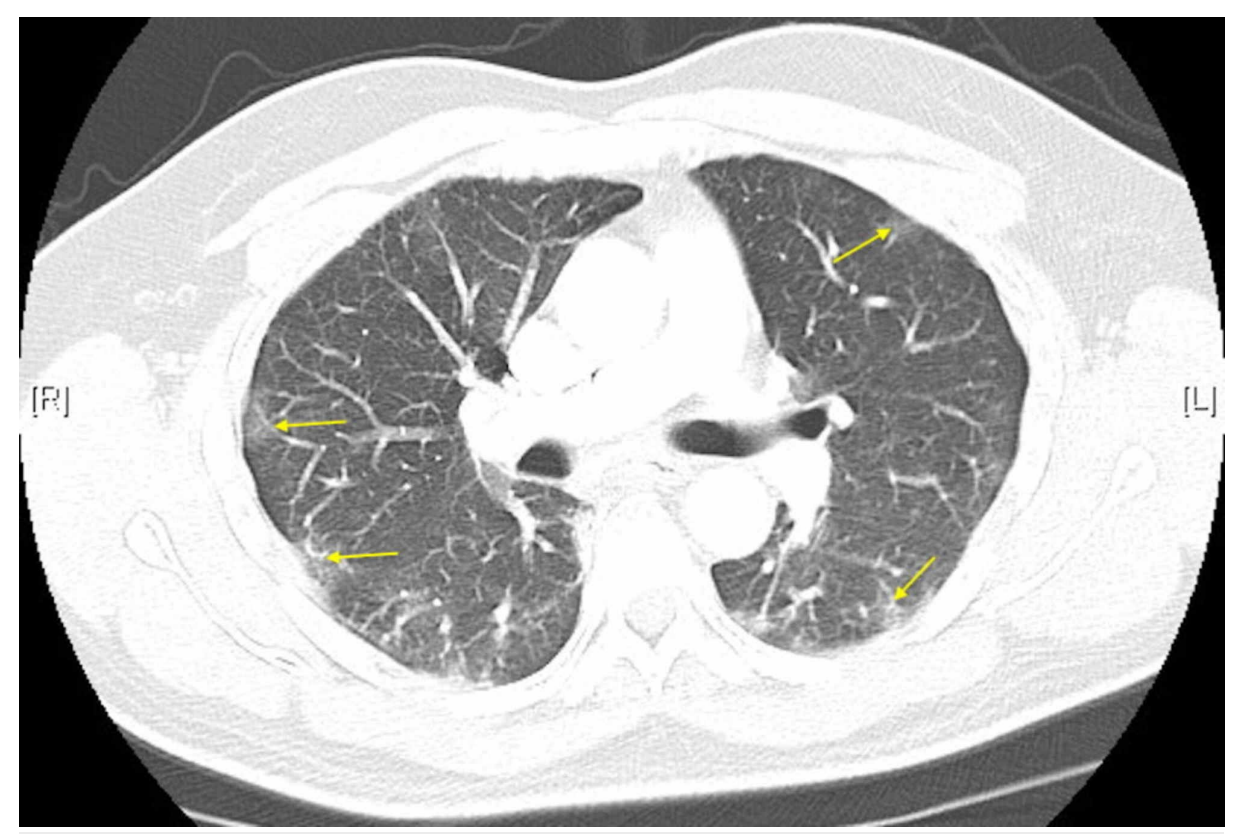

FIGURE 2: Chest CT demonstrating bilateral ground glass opacities 


\section{Cureus}

He appeared well throughout the remainder of his stay in the ED with stable vitals. The patient was ambulated in the ED with no decrease in oxygen saturation noted. Although the patient had imaging findings typical of COVID-19 pneumonia and a corroborating history of present illness, the patient was nonetheless treated with $500 \mathrm{mg}$ intravenous azithromycin and $1 \mathrm{~g}$ of ceftriaxone as empiric treatment for community-acquired pneumonia. Given that the patient had no other comorbidities, and wanted to go home, he was discharged home with a course of oral antibiotics. During a telephone follow-up three days after being discharged, the patient reported that he continued to feel better while at home.

\section{Discussion}

CT scans have recently been embraced by EDs where RT-PCR and sequencing are not available, possibility due to a lack of availability [5]. Moreover, its high sensitivity in diagnosing COVID-19, $97 \%$ in a study of 1,014 patients, makes it a useful diagnostic tool even when RT-PCR is available, as the same study reported a five-day interval between initial negative and initial positive RT-PCR tests [6].

Although there exists some variability in CT scan findings across patient cases, GGO remains the main CT feature, with involvement in multiple lobes in most cases [7]. Bilateral involvement and peripheral distribution are typically observed, and consolidation is observed in some cases as well [5].

COVID-19 presentation typically also includes some form of respiratory distress, with common clinical features across the pandemic including fever, coughs and sore throat, headaches, myalgia, and shortness of breath [8]. Myalgia, dyspnea, expectoration, diarrhea, nausea and vomiting, and general gastrointestinal involvement have also been reported as common clinical symptoms in patients [9]. Laboratory results often show leukopenia and elevated D-dimers [10].

The case presented shows a patient notable for some of the common COVID-19 symptoms, showing on-andoff fevers and chills, shortness of breath, elevated D-dimers, and leukopenia, but no additional organ system involvement (including gastrointestinal), no dyspnea or acute respiratory distress, no myalgia, and a lack of other common symptoms (Figure 3). 


\section{Cureus}

\section{TYPICAL \\ SYMPTOMS OF COVID-19}

\section{PRESENTATION}

- Respiratory distress (dyspnea, shortness of breath, hypoxia with movement)

- Fever and headaches

- Gastrointestinal symptoms (nausea, vomiting, diarrhea)

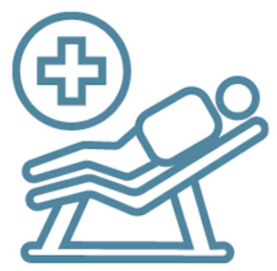

\section{IMAGING}

- Ground glass opacities

- Bilateral involvement

- Peripheral distribution

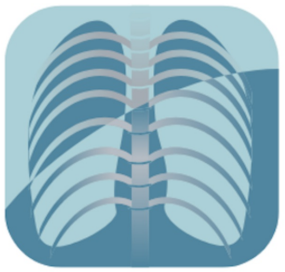

\section{LABORATORY}

- Elevated D-Dimers

- Leukopenia

- Lymphopenia

- Prolonged prothrombin time (PT)

- Elevated lactate dehydrogenase (LDH)

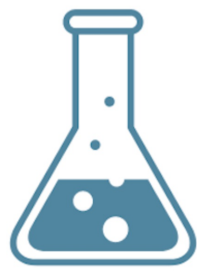

FIGURE 3: Common findings associated with COVID-19 pneumonia

The patients x-ray and CT scans reveal multifocal GGO. Nonetheless, the patent was able to go home from the ED. On three-day telephone follow-up, the patient was still managing okay at home.

Although this case shows typical attributes of COVID-19 viral pneumonia, the overall severity of this case is lower than literature tends to suggest to date. While many factors are at play in the lower ratio of COVID-19 related deaths to cases, it is entirely possible that as new cases are encountered in less at-risk populations, the course of the disease will be more akin to what we have observed. Although the one case observed here provides only a single point of anecdotal evidence, taken in combination with the lowering ratio of deaths to cases it may suggest the importance of observing the characteristics and symptoms of COVID-19 over the course of the pandemic and determining whether, since the initial outbreak and arrival in the country, a significant change in the lethality of the disease exists and for what reasons. 
This more benign form of pneumonia may reflect a lower virulence of the current COVID-19 strain, or may reflect patient factors. Some reported risk factors through to contribute to COVID-19 morbidity include obesity, smoking, alcoholism, and poor overall health. Our patient had a normal BMI, did not smoke cigarettes or drink alcohol, and had no medical history. Thus, despite his age of 51 years, he was overall healthy and presumably able to combat the virus well.

\section{Conclusions}

This case reports on a seemingly less virulent COVID-19 pneumonia, with a more benign course compared to the COVID-19 pneumonias we saw several months ago. This may be due to characteristic associated with the virus itself including mutation, or may have to do with patient factors. It is important to recognize various patterns in this evolving pandemic.

\section{Additional Information \\ Disclosures}

Human subjects: Consent was obtained by all participants in this study. HCA Centralized Algorithms for Research Rules on IRB Exemptions (CARRIE)/ IRB manager issued approval 2020-527. Based on the information provided and attested as true, the research plan described does not require IRB oversight. This is because you are either a) not engaging in research with human subjects as defined by federal regulations; $b$ ) engaging in research with human subjects deemed excluded from IRB oversight per 45CFR46.102(l) OR c) engaging in research with sufficient human subject protections in the design to meet one or more IRB exemption criteria set forth in 45CFR46.104. Conflicts of interest: In compliance with the ICMJE uniform disclosure form, all authors declare the following: Payment/services info: All authors have declared that no financial support was received from any organization for the submitted work. Financial relationships: All authors have declared that they have no financial relationships at present or within the previous three years with any organizations that might have an interest in the submitted work. Other relationships: All authors have declared that there are no other relationships or activities that could appear to have influenced the submitted work.

\section{Acknowledgements}

This research was supported (in whole or in part) by HCA Healthcare and/or an HCA Healthcare affiliated entity. The views expressed in this publication represent those of the author(s) and do not necessarily represent the official views of HCA Healthcare or any of its affiliated entities.

\section{References}

1. WHO statement regarding cluster of pneumonia cases in Wuhan, China . (2020). Accessed: July 9, 2020: https://www.who.int/china/news/detail/09-01-2020-who-statement-regarding-cluster-of-pneumonia-casesin-wuhan-china.

2. Holshue ML, DeBolt C, Lindquist S, et al.: First case of 2019 novel coronavirus in the United States . N Engl J Med. 2020, 382:929-936. 10.1056/NEJMoa2001191

3. Coronavirus disease 2019 (COVID-19). Cases in the U.S . (2020). Accessed: July 9, 2020: https://www.cdc.gov/coronavirus/2019-ncov/cases-updates/cases-in-us.html.

4. Amid COVID-19 resurgence, fewer deaths. Dr. Fauci explains why . (2020). Accessed: July 9, 2020: https://www.ama-assn.org/delivering-care/public-health/amid-covid-19-resurgence-fewer-deaths-dr-fauciexplains-why.

5. Hani C, Trieu NH, Saab I, Dangeard S, Bennani S, Chassagnon G, Revel M-P: COVID-19 pneumonia: a review of typical CT findings and differential diagnosis. Diagn Interv Imaging. 2020, 101:263-268. 10.1016/j.diii.2020.03.014

6. Ai T, Yang Z, Hou H, et al.: Correlation of chest CT and RT-PCR testing for coronavirus disease 2019 (COVID-19) in China: a report of 1014 cases. Radiology. 2020, 296:E32-E40. 10.1148/radiol.2020200642

7. Lin X, Gong Z, Xiao Z, Xiong J, Fan B, Liu J: Novel coronavirus pneumonia outbreak in 2019: computed tomographic findings in two cases. Korean J Radiol. 2020, 21:365-368. 10.3348/kjr.2020.0078

8. Singhal T: A review of coronavirus disease-2019 (COVID-19). Indian J Pediatr. 2020, 87:281-286 10.1007/s12098-020-03263-6

9. Li L-Q, Huang T, Wang Y-Q, et al.: COVID-19 patients' clinical characteristics, discharge rate, and fatality rate of meta-analysis. J Med Virol. 2020, 92:577-583. 10.1002/jmv.25757

10. Zhu J, Ji P, Pang J, et al.: Clinical characteristics of 3,062 COVID-19 patients: a meta-analysis [Epub ahead of print]. J Med Virol. 2020, 10.1002/jmv.25884 